\title{
Generating and characterizing the anti-human CD45 monoclonal antibody
}

\author{
Giang Huong Ta, Huy Quoc Nguyen, Quan Dang Nguyen*
}

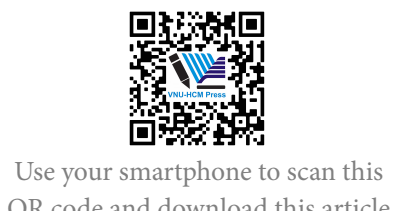

QR code and download this article
Medical Biotechnology Department, Biotechnology Center of Ho Chi Minh City, 2374 Highway 1, Trung My Tay Ward, District 12, Ho Chi Minh City

\section{Correspondence}

Quan Dang Nguyen, Medical Biotechnology Department, Biotechnology Center of Ho Chi Minh City, 2374 Highway 1, Trung My Tay Ward, District 12, Ho Chi Minh City

Email: ndquan.snn@tphcm.gov.vn

History

- Received: 2020-06-15

- Accepted: 2020-08-19

- Published: 2020-09-04

DOI : 10.32508/stdj.v23i3.2409

\section{Check for updates}

\section{Copyright}

(c) VNU-HCM Press. This is an openaccess article distributed under the terms of the Creative Commons Attribution 4.0 International license.

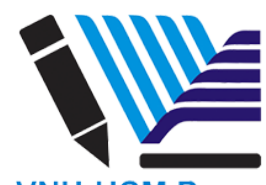

VNU-HCM Press

\begin{abstract}
Introduction: CD45 is a common marker of leukocytes. Anti-human CD45 monoclonal antibody (MAb) has been used widely in diagnosing and monitoring hematologic diseases. The aim of this study was to generate an anti-human CD45 MAb, which can be used in research and diagnosis. Methods: Recombinant human CD45RO antigen was expressed from E. coli BL21 (DE3), purified and analyzed by SDS-PAGE and Western blotting. The purified CD45RO antigen was used to immunize Balb/c mice. Spleen cells from immunized mouse were collected and fused with P3X63Ag8.653 myeloma cells to form hybridoma. Anti-CD45 antibody-secreting capacity of hybridoma clones was evaluated by ELISA assay. Anti-CD45 MAb from the culture supernatant of the chosen hybridoma clone was purified by affinity chromatography. The MAb was characterized the biochemical characteristics and biological activity. Results: Recombinant human CD45RO antigen was expressed and purified from E.coli BL21 (DE3). Injection of purified CD45RO antigen provoked the immune response in Balb/c mice. Hybridoma clones were generated successfully by the fusion of spleen cells from the selected immunized-mouse and myeloma cells. Among these hybridoma clones, one with the highest yield of MAb production was identified. The isotype of the anti-CD45 MAb created in this work is IgG2b, while its the light chain is kappa (k) type. The affinity of this MAb with CD45RO antigen is high with $\mathrm{K}_{d}$ value at the picomolar level. The anti-CD45 MAb can interact with CD45 naturally expressed on the surface of Jurkat cells in Western blotting and fluorescent immuno-staining assay. Conclusion: We have developed successfully an anti-human CD45 MAb using hybridoma technology, which can recognize CD45 in ELISA, Western blotting, and fluorescent immuno-staining analysis. Although further investigations are necessary, obviously, our anti-human CD45 MAb is potential for research and diagnosis applications.
\end{abstract}

Key words: CD45, anti-human CD45 antibody, monoclonal antibody

\section{INTRODUCTION}

CD45 is a common marker on the surface of leukocytes. In mammals, gene encoding CD45 is ptprc, which contains 35 well-characterized exons ${ }^{1}$. The exons 4, 5, 6 of ptprc mRNA are alternatively spliced to create a variety of CD45 isoforms at different molecular weights from $180 \mathrm{kDa}$ to $220 \mathrm{kDa}$. CD $45 \mathrm{RABC}$ is the full-length isoform which contains region encoded by exons $4,5,6$, and CD45RO is the shortest isoform, which does not include the region encoded by these exons ${ }^{1,2}$. Therefore, the CD45RO is the common structure presenting on all CD45 isoforms. Depending on the cell type and developing stage, different CD45 isoforms are expressed on the cell surface. B lymphocytes express CD45RABC isoform; in contrast, naive T lymphocytes express CD45RA isoform while effector $\mathrm{T}$ lymphocytes express the shortest CD45RO isoform ${ }^{2,3}$.

CD45 is a fundamental and important marker for defining the immunophenotyping of leukocyte populations in normal conditions, hematological malig- nancies, and other hematologic diseases. In combination with other markers, CD45 is helpful in identifying and distinguishing a leukocyte population (such as B cells, T cells, monocytes, granulocytes,...) from other cell populations in flow cytometry analysis $^{4,5}$. Thus, anti-CD45 MAb is indispensable for immunophenotyping practices in research as well as in diagnosis. Up to now, there is no anti-CD45 MAb developed in Vietnam, and all anti-CD45 MAbs for satisfying the domestic demands are imported. Aim of this work was to create an anti-human CD45 MAb, which would be potential for research and diagnosis applications. To make the anti-CD45 MAb being able to recognize all isoforms of human CD45, we chose the extracellular region of human CD45RO as the antigen to develop the target antibody. The produced anti-human CD45 MAb was characterized by the biochemical properties and the antigen interacting activity in ELISA, Western blotting, and fluorescent immunostaining assays. 


\section{MATERIALS - METHODS}

\section{Expression and purification of CD45RO antigen}

E. coli BL21 (DE3) was transfected by pET21a(+) plasmid encoding $\mathrm{N}$ - and C-terminal His-tag human CD45RO extracellular region or empty plasmid for control. The sequence of the huCD45 gene was from NCBI Genebank (code NM_080921.3). Transfected E.coli was cultured in LB medium plus Kanamycine $(50 \mu \mathrm{g} / \mathrm{ml})$ at $37^{\circ} \mathrm{C}, 250 \mathrm{rpm}$, and induced for protein expression by IPTG $1 \mathrm{mM}$ (Biobasic, Ontario and Amherst, NY) for 6 hours. The E.coli biomass was harvested by centrifugation at 6,000rpm for $5 \mathrm{~min}$ utes and lysed by sonication to collect the total lysate fragment. From total lysate fragment, the pellet and supernatant lysate fragments were separated by centrifugation at $13,000 \mathrm{rpm}, 4^{\circ} \mathrm{C}$, for 10 minutes. The recombinant $\mathrm{CD} 45 \mathrm{RO}$ protein in cell lysate fragments was identified by SDS-PAGE and Western blotting using the MEM-28 anti-CD45 antibody (ab8216 - Abcam, Cambridge, UK).

Recombinant CD45RO protein from pellet fragment was dissolved by urea $8 \mathrm{M}$ solution and was purified by Histrap HP 5ml column (GE Healthcare, Chicago, IL) on AKTA purifier (GE Healthcare, Chicago, IL). The purified CD45RO protein was analyzed by SDS-PAGE with silver staining, and the purity of the protein was evaluated by ImageJ software.

\section{Generation of the hybridoma secreting the anti-human CD45 antibody}

Three 6-8 week old $B a l b / c$ female mice were immunized subcutaneously three times with $50 \mu \mathrm{g}$ of purified CD45RO emulsified in Complete Freund's Adjuvant (Sigma Aldrich, St Louis, MO) at day 1, 15, 30 and boosted at day 60 with antigen in Incomplete Freund's Adjuvant (Sigma Aldrich, St Louis, MO). Serum samples from immunized mice were collected on day 45 for anti-CD45RO antibody titer testing by ELISA. At day 63, spleen cells from the highest immuneresponding mouse were obtained and fused with P3X63Ag8.653 myeloma cells at ratio 5:1 to create hybridoma using PEG 1500 (Sigma Aldrich, St Louis, MO). The hybridoma cells were diluted in $200 \mathrm{ml}$ of HAT medium (hypoxanthine, aminopterin, and thymidine) (Sigma Aldrich, St Louis, MO) supplemented with 20\% FBS (Sigma Aldrich, St Louis, MO) and were cultured in 20 of 96 -well plates $(100 \mu \mathrm{l} /$ well). After 15 days, the presence of anti-CD45RO antibody in culture supernatants from wells containing proliferating cells was determined by ELISA assay.
Hybridoma clones secreted anti-CD45RO antibody at the highest concentrations were kept expanding in HAT medium 20\% FBS. Clones proliferating well in selective medium and producing anti-CD45RO antibody stably were subcloned by limiting dilution method to make single clones of hybridoma. Single hybridoma clones that survived after many cycles of culture were investigated the proliferation and antibody production in RPMI medium (Gibco, Carlsbad, CA) $10 \%$ FBS to identify the best single clone for further experiments.

The animal experiments were approved by the Biotechnology Center of Ho Chi Minh City and were performed according to the guideline on animal experiments.

\section{Detection of anti-CD45 antibody by ELISA assay}

ELISA plate was coated with $50 \mu \mathrm{l}$ of CD45RO antigen solution $(5 \mu \mathrm{g} / \mathrm{ml})$ per well and incubated at $4^{0} \mathrm{C}$, overnight. After washing and blocking steps, $100 \mu \mathrm{l}$ of hybridoma culture supernatants were added to wells and incubated for 2 hours at room temperature. After washing the plate, $50 \mu \mathrm{l}$ of goat anti-mouse IgG antibody HRP conjugated (62-6520- Thermo Scientific, Waltham, MA) solution (1:1000) was added to each well and incubated 90 minutes at room temperature. TMB substrate (Sigma Aldrich, St Louis, MO) was added to wells after thoroughly washing the plate. The plate was incubated 30 minutes in the dark, and $\mathrm{OD}_{450 \mathrm{~nm}}$ values of wells were measured after the addition of $25 \mu \mathrm{l}$ of $\mathrm{H}_{2} \mathrm{SO}_{4} 2 \mathrm{M}$ solution to stop the reaction.

\section{Expression and purification of anti-CD45 MAb}

Selected hybridoma clone was cultured in serum-free medium Hybridoma -SFM (12300067-Gibco, Carlsbad, CA) at a density of $2 \times 10^{6}$ cells $/ \mathrm{ml}$ in a humidified incubator at $37^{\circ} \mathrm{C}, 5 \% \mathrm{CO}_{2}$. After 4 days, culture supernatant was harvested. Anti-CD45 MAb in the culture supernatant was purified by affinity chromatography using Protein G HP $5 \mathrm{ml}$ column (GE Healthcare, Chicago, IL) on AKTA purifier. Buffers used in the MAb purification included: binding buffer $20 \mathrm{mM}$ sodium phosphate, $\mathrm{pH} 7.4$; elution buffer: $0.1 \mathrm{M}$ glycine $-\mathrm{HCl}, \mathrm{pH} 2.7$; neutralization buffer: $1 \mathrm{M}$ Tris $-\mathrm{HCl}, \mathrm{pH}$ 9. Purified MAb was stored in PBS 1X, at $4^{\circ} \mathrm{C}$. 
Evaluation of the interaction affinity between anti-CD45 MAb and recombinant CD45RO

The interaction affinity, $\mathrm{K}_{d}$ value, was evaluated by ELISA assay in which wells were coated by recombinant CD45RO antigen at four different concentrations $(5,2.5,1.25,0.625 \mu \mathrm{g} / \mathrm{ml})$. At each concentration of antigen, 3 -fold serial dilutions starting from $70,000 \mathrm{ng} / \mathrm{ml}$ of the purified anti-CD45 MAb were added. The ELISA assay was performed as described above. Corresponding to each antigen concentration, the curve of correlation between OD450nm values and antibody concentrations was established. Basing on these curves, the $\mathrm{K}_{d}$ values were determined by formula:

$$
\mathrm{K}_{\mathrm{aff}}=\frac{\mathrm{n}-1}{2\left(\mathrm{n}\left[\mathrm{Ab}^{\prime}\right]-[\mathrm{Ab}]\right)} \quad \mathrm{K}_{\mathrm{d}}=\frac{1}{\mathrm{~K}_{\mathrm{aff}}}
$$

$\mathrm{K}_{a f f}$ : affinity constant; $\mathrm{K}_{d}$ : dissociation constant; $\mathrm{n}$ : antigen dilution factor ([Ag]/[Ag']); $[\mathrm{Ab}]$ and $\left[\mathrm{Ab}^{\prime}\right]$ : antibody concentrations giving $\mathrm{OD}_{\frac{1}{2} \max }$ values which are equal a half of the maximum OेD values in the wells coated with antigen at the concentration of $[\mathrm{Ag}]$ and $\left[\mathrm{Ag}^{\prime}\right]^{6}$.

With 4 different antigen concentrations ([Ag]), 4 antibody concentrations $([\mathrm{Ab}])$ providing $\mathrm{OD}_{\frac{1}{2} \max }$ values were determined. From $4[\mathrm{Ab}]$ values, $6 \mathrm{~K}_{a f f}$ values were calculated by 6 pair combinations of $[\mathrm{Ab}]$ values, and $6 \mathrm{~K}_{d}$ values were identified consequently. The final $\mathrm{K}_{d}$ value was average $\pm \mathrm{SD}$ of the $6 \mathrm{~K}_{d}$ values above. $\mathrm{K}_{d}$ value was calculated with $[\mathrm{Ab}]$ at molar concentrations which was converted from w/v concentration as following: molar concentration $(\mathrm{nM})=$ w/v concentration ( $\mathrm{ng} / \mathrm{ml}$ )/150 (MW of IgG antibody in $\mathrm{KDa})$.

\section{Western blotting using anti-CD45 MAb}

$20 \mu \mathrm{g}$ protein from $E$. coli which expressed recombinant CD45RO, Jurkat and HEK293 cell lysates were separated by SDS-PAGE and transferred to PVDF membrane. The membrane was stained with the antiCD45 MAb or commercial M-28 anti-CD45 MAb solution (1:2000 dilution from $1 \mathrm{mg} / \mathrm{ml}$ stock of antibody) and then with the secondary anti-mouse IgG HRP conjugated (62-6520- Thermo Scientific, Waltham, MA). The signal on the membrane was developed by the DAB substrate (Sigma Aldrich, St Louis, MO).

Fluorescent immunostaining with antiCD45 MAb

$5 \times 10^{5}$ Jurkat and HEK 293 cells were incubated in $100 \mu \mathrm{l}$ of cold PBS $1 \%$ BSA containing $10 \mu \mathrm{g} / \mathrm{ml}$ of
anti-CD45 MAb or MEM-28 anti-CD45 antibody for 1 hour, at room temperature, shake gently. Cells were washed by cold PBS $1 \%$ BSA and then incubated in $100 \mu \mathrm{l}$ of cold PBS $1 \%$ BSA containing $10 \mu \mathrm{g} / \mathrm{ml}$ of goat anti-mouse IgG FITC conjugated (sc-2010 - Santa Cruz Biotechnology, Dallas, TX) for 1 hour, in the dark, at room temperature. Cells were washed by cold PBS 1\% BSA. Finally, cells were fixed with $100 \mu$ l of paraformaldehyde $1 \%$ and observed under a fluorescent microscope (Carl Zeiss, Oberkochen, Germany).

\section{RESULTS}

\section{Expressing and purifying the recombinant CD45RO}

SDS-PAGE analysis of the cell lysate from E. coli transfected by plasmid encoding human CD45RO extracellular region showed the overexpression of a $\sim 55$ $\mathrm{kDa}$ protein in the total and pellet cell lysate fragments, but not in the supernatant fragment. This protein band was not seen in the total cell lysate fragment of $E$. coli containing an empty pET vector (Figure 1 A). Thus, this overexpressed protein might be the target of CD45RO protein. As expected, the Western blotting using commercial MEM-28 anti-CD45 antibody confirmed that the $55 \mathrm{kDa}$ protein band is the recombinant CD45RO (Figure $1 \mathrm{~B}$ ). Hence, the recombinant CD45RO protein was expressed successfully from $E$. coli as an insoluble inclusion body form. The insoluble recombinant CD45RO protein in the inclusion body was dissolved in urea $8 \mathrm{M}$ solution and was purified by affinity chromatography using Histrap HP $5 \mathrm{ml}$ (GE Healthcare, Chicago, IL). SDSPAGE with silver staining indicated that recombinant CD45RO protein was purified well with the purity of more than $90 \%$ according to ImageJ software analysis (Figure 1 C). The purified recombinant CD45RO was used to immunize mice.

\section{Immunizing Balb/c mice, generating and screening the hybridoma secreting anti- CD45RO MAb}

Three Balb/c mice immunized by recombinant CD45RO provoked good immune responses with the serum anti-CD45 antibody titer in range of $1 / 25,600$ to $1 / 51,200$ dilution (data not shown). The highest immune responding mouse was chosen to collect spleen cells for hybridoma formation. From 20 of 96-well plates (1,920 wells) of hybridoma culture in HAT medium, we identified 196 wells containing proliferating cells. The presence of anti-CD45RO antibody in 196 supernatants was investigated by 


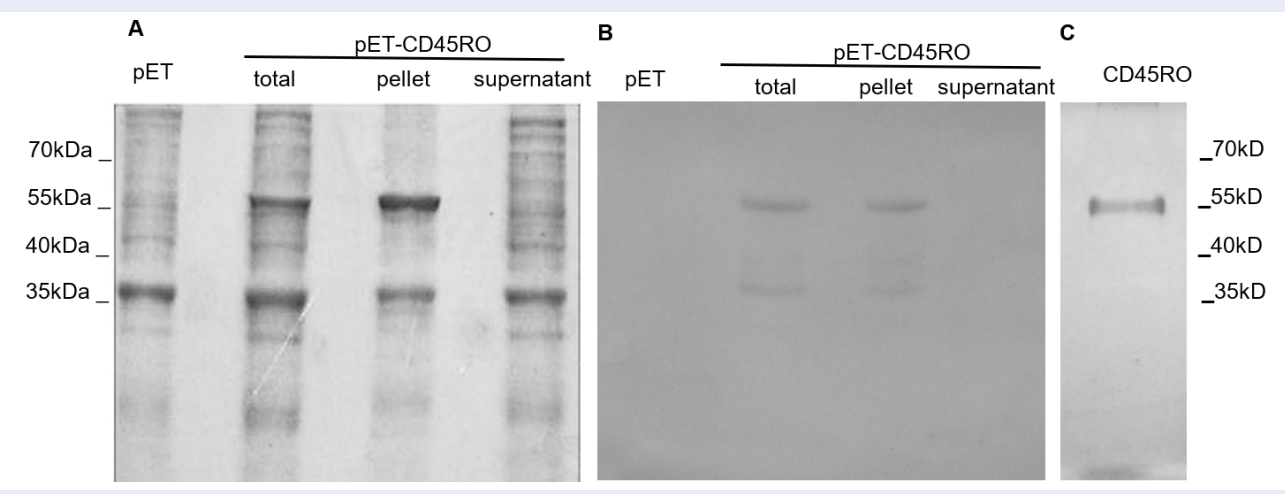

Figure 1: Expression of recombinant CD45RO antigen from E.coli BL21 (DE3). (A): SDS-PAGE analysis of cell lysatesof $E$. coli containing empty vector ( $\mathrm{pET}$ ) and CD45RO-encoding vector (pET-CD45RO); (B): Western blotting analysis of $E$. coli cell lysates using commercial MEM-28 anti-CD45 MAb; (C): SDS-PAGE analysis and silver staining of purified recombinant CD45RO.

ELISA using recombinant CD45RO as an antigen. Among 196 viable hybridoma clones, we have determined 3 clones that secreted anti-CD45RO antibody at the highest yields (data not shown). From the 3 chosen clones, through a process of culturing in selective HAT medium, expanding and subcloning, we harvested 3 single hybridoma clones named 15B10-E5, 16E8-F2, 16E8-E2 which could survive and produce the antibody stably.

The proliferation and anti-CD45RO antibody secretion capacity of single clones were investigated. The results showed that cell density and antibody concentration of these clones reached the highest on the third day of culture and decreased in the following days (Figure $2 \mathrm{~A}$ and $\mathrm{B}$ ). Among the three single hybridoma clones, 16E8-F2 clone exhibited the highest yield at both cell density and antibody secretion. Therefore, we chose 16E8-F2 clone for the following experiments.

\section{The biochemical characteristics of 16E8-F2 anti-CD45 MAb}

16E8-F2 hybridoma clone was cultured in serum-free medium for antibody expression, and the anti-CD45 $\mathrm{MAb}$ in the culture supernatant was purified by affinity chromatography. Purified 16E8-F2 anti-CD45 MAb was simultaneously evaluated by SDS-PAGE in reducing and non-reducing conditions. In reducing condition, the antibody sample was visualized as two bands of $\sim 55 \mathrm{kDa}$ and $\sim 25 \mathrm{kDa}$ corresponding with the heavy chain and light chain of the antibody. While in non-reducing conditions, the 16E8-F2 anti-CD45 $\mathrm{MAb}$ appeared as one band of $\sim 170 \mathrm{kDa}$. This implies that anti-CD45 MAb expressed from 16E8-F2 clone might be secreted into the culture supernatant as an intact antibody with 2 heavy chains and 2 light chains (Figure $3 \mathrm{~A}$ ). The Western blotting analysis using an anti-mouse IgG $\mathrm{H}+\mathrm{L}$ antibody (62-6520 Thermo Fisher) revealed that these bands are the fragments of the mouse IgG antibody (Figure 3 B). Using the isotyping kit (11493027001- Roche), we have identified that the 16E8-F2 anti-CD45 MAb created in this work is $\operatorname{IgG} 2 \mathrm{~b}$, and its light chain is kappa $(\mathrm{k})$ type (Figure $3 \mathrm{C}$ ).

\section{The biological activity of 16E8-F2 anti- CD45 MAb}

\section{The affinity with CD45RO antigen}

Antigen affinity is an important characteristic which demonstrates the quality of an antibody. The affinity of antibody and antigen is evaluated via the dissociation constant $\mathrm{K}_{d}$ value, the lower $\mathrm{K}_{d}$ value, the higher binding affinity of antibody and its target. In this work, the $\mathrm{K}_{d}$ value of 16E8-F2 anti-CD45 antibody with recombinant $\mathrm{CD} 45 \mathrm{RO}$ antigen was determined using the method of Beatty et al. $1987^{6}$. In which, ELISA assay was performed with four different coated CD45RO antigen concentrations interacting with 3fold serial diluted 16E8-F2 anti-CD45 MAb concentrations. The correlation curves of $\mathrm{OD}_{450 \mathrm{~nm}}$ values and the logarithm base 3 of antibody concentrations $(\mathrm{ng} / \mathrm{ml})$ corresponding to each antigen concentration were established (Figure 4). Relying on these curves, 4 antibody concentrations providing $\mathrm{OD}_{\frac{1}{2} \max }$ values and consequently, $6 \mathrm{~K}_{d}$ values were calculated. Then, the final $\mathrm{K}_{d}$ value of the interaction between 16E8F2 anti-CD45 MAb and CD45RO antigen was determined of $7.13 \pm 2.26 \times 10^{-10} \mathrm{M}$. 
A

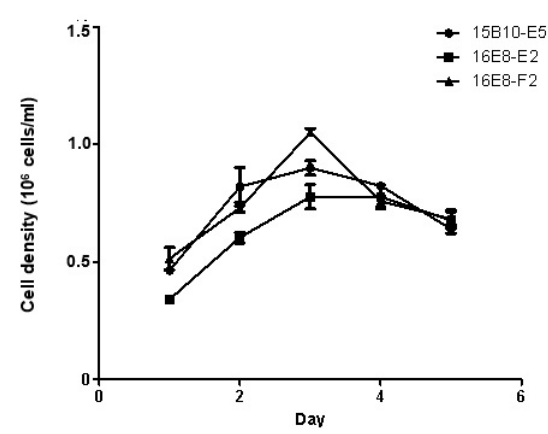

B

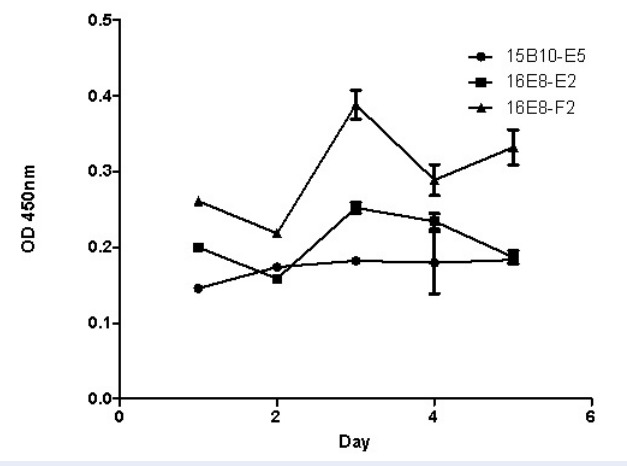

Figure 2: Proliferation and anti-CD45RO antibody secretion capacity of single hybridoma clones. (A): Cell proliferation in culture; (B): Antibody secretion capacity evaluated by ELISA. Values were represented as mean \pm $\mathrm{SD}(\mathrm{n}=3)$

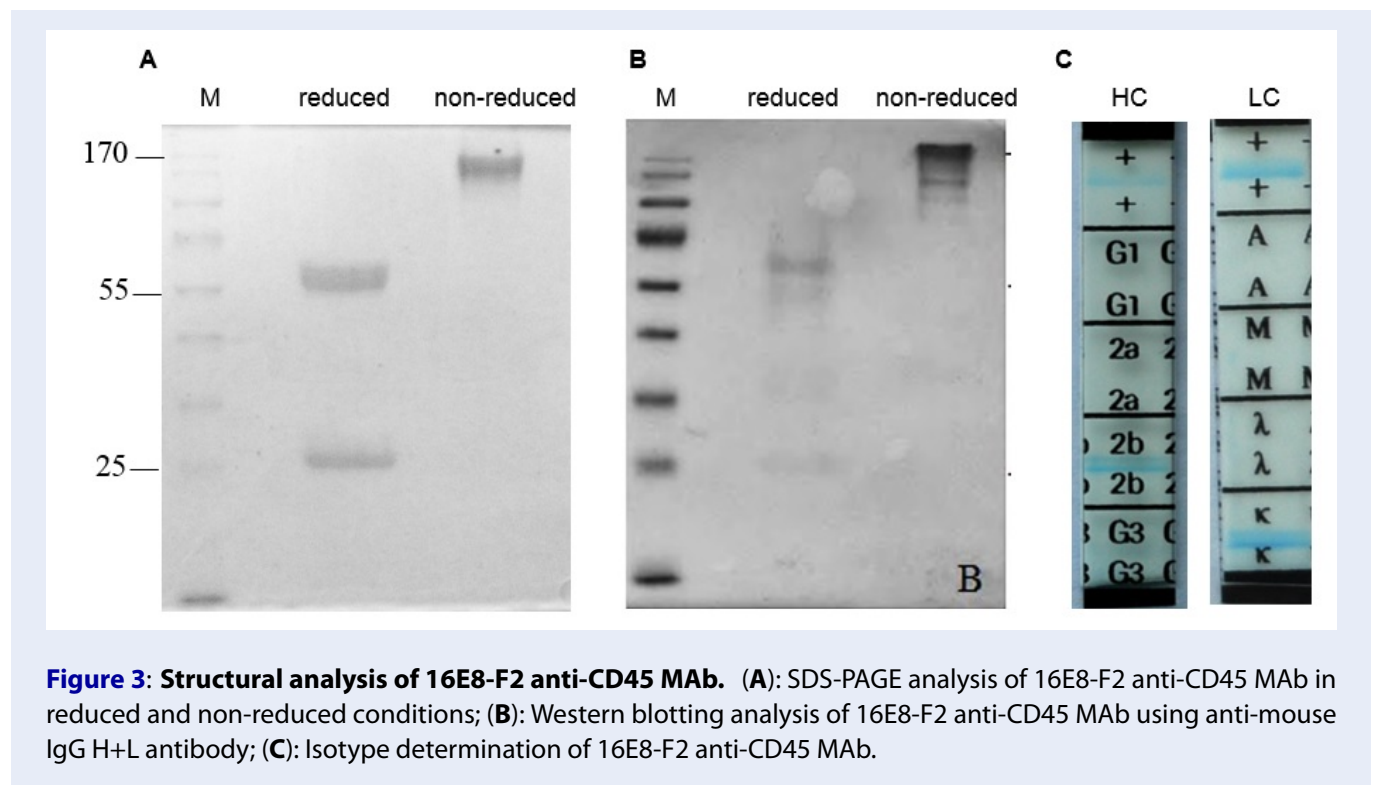

The activity of 16E8-F2 anti-CD45 MAb in Western blot

The performance of 16E8-F2 anti-CD45 MAb in recognition of recombinant antigen and natural CD45 expressed in lymphocytes was evaluated by Western blotting. With the cell lysate of E. coli expressing CD45RO, the Western blotting analysis showed that 16E8-F2 anti-CD45 MAb and commercial MEM-28 anti-CD45 antibody recognize specifically a protein band of $\sim 55 \mathrm{kDa}$ which corresponding to the recombinant CD45RO extracellular region. In contrast, with lysate of E. coli non-induced CD45RO expression, both antibodies gave no signal or very weak signal (Figure $5 \mathrm{~A}$ ). In addition, cell lysates of $\mathrm{T}$ cell line
Jurkat and epithelial cell line HEK293 were analyzed by SDS-PAGE and Western blotting using 16E8-F2 anti-CD45 MAb and MEM-28 antibody. The result reveals that both antibodies detected a double band of protein at above $170 \mathrm{kDa}$ in Jurkat cell lysate, but not in the control sample of HEK293 cell lysate (Figure 5 C and D). Jurkat cells were known to express CD45RO $(180 \mathrm{kDa})$ and CD45RA $(190 \mathrm{kDa})$ on their surface ${ }^{7}$; hence the double band seen in the Western blotting analysis may be these 2 CD45 isoforms naturally expressed by Jurkat cells. It manifests that our antiCD45 MAb can recognize CD45 expressed by leukocytes in the Western blotting analysis similar to that the commercial MEM-28 anti-CD45 antibody does. 


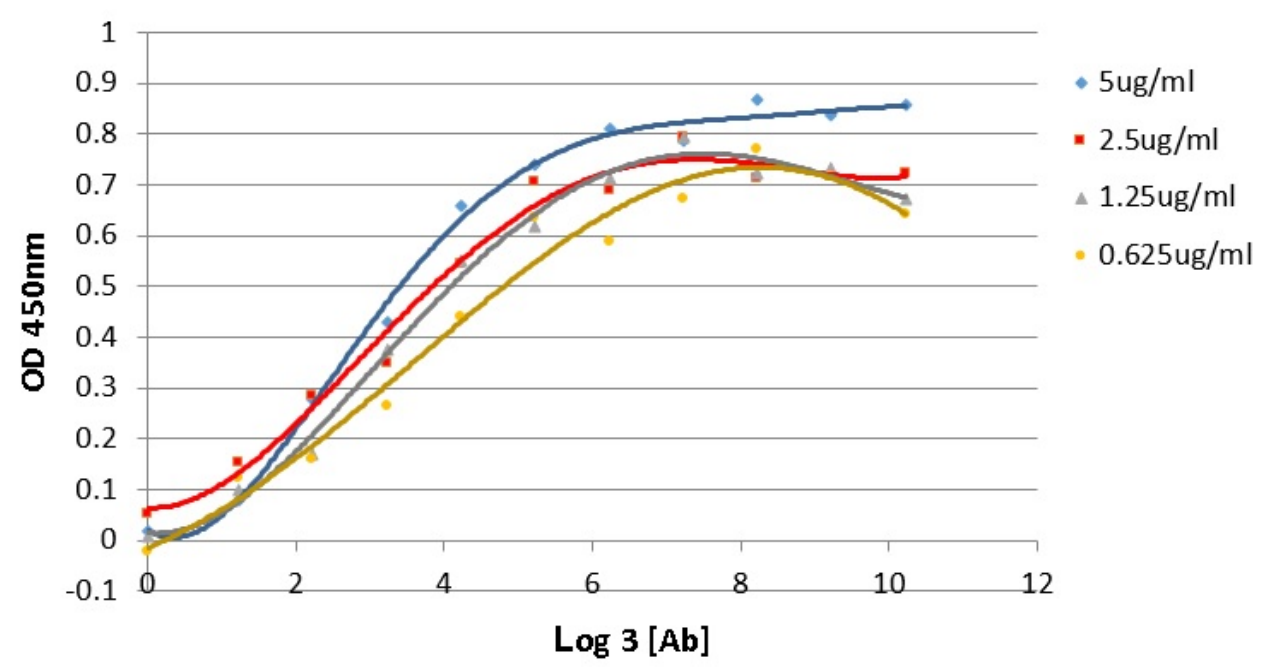

Figure 4: Determination of the antigen affinity of anti-CD45 MAb by ELISA. The correlation curves of $\mathrm{OD}_{450 \mathrm{~nm}}$ values and the logarithm base 3 of 16E8-F2 anti-CD45 MAb concentrations (3-fold serial dilution from $70,000 \mathrm{ng} / \mathrm{ml}$ ) corresponding to different antigen concentrations were established. Basing on these curves the $\mathrm{K}_{d}$ value was calculated.

Taken together, the 16E8-F2 anti-CD45 MAb generated in this work can be used to detect its antigen in Western blotting assay.

The activity of 16E8-F2 anti-CD45 MAb in fluorescent immuno-staining

We next addressed the question of whether 16E8-F2 anti-CD45 MAb can work in a fluorescent immunostaining assay for the detection of non-denatured CD45 expressed on the cell surface. 16E8-F2 antiCD45 MAb was utilized to stain Jurkat T cells, which express CD45 on their surface and HEK293 cells as a negative control. In parallel, MEM-28 anti-CD45 MAb was also used to stain Jurkat T cells for comparison. All cell samples were incubated with an antimouse IgG-FITC secondary antibody before visualized under the fluorescent microscope. As expected, the fluorescent signal was observed from Jurkat cells immuno-stained by both 16E8-F2 and MEM-28 antiCD45 MAb. On the other hand, no fluorescent signal was seen from HEK293 cells stained by 16E8-F2 anti-CD45 MAb (Figure 6). The result demonstrated that our 16E8-F2 anti-CD45 MAb could interact with natural CD45 antigen on the lymphocyte surface in the fluorescent immuno-staining assay. Interestingly, this antibody may not cross-react with surface proteins on CD45 negative cells.

\section{DISCUSSION}

CD45 is a common marker of leukocytes, and antiCD45 MAb is used widely in the diagnosis of blood diseases such as leukemia, lymphoma, and other hematologic diseases. This project was carried out to generate an anti-CD45 MAb satisfying the domestic demands. We have created 16E8-F2 anti-CD45 MAb, which is $\operatorname{IgG} 2 \mathrm{~b} / \mathrm{k}$ isotype and is produced in the full structure with two light chains and two heavy chains. Antigen affinity is one of the most important factors of antibody quality, which is evaluated by $\mathrm{K}_{d}$ value, the smaller $\mathrm{K}_{d}$ value the higher antigen affinity $^{6}$. Generally, antibodies which have $\mathrm{K}_{d}$ values lower than nanomolar level are ranked as high antigen affinity. With the $\mathrm{K}_{d}$ value at the picomolar level $\left(7.13 \pm 2.26 \times 10^{-10}\right.$ molar) determined in this study, 16E8-F2 anti-CD45 MAb has a high affinity with its antigen CD45RO. However, with current data, we do not compare the antigen affinity of our 16E8-F2 antiCD45 MAb to that of other commercial antibodies because the investigations were not performed in the same condition.

Western blotting analysis of the CD45RO-expressing E.coli lysate proved that 16E8-F2 anti-CD45 MAb could detect specifically the target recombinant CD45RO protein, and it does not cross-react with any other protein in total E. coli cell lysate. With lysate of $E$. coli non-induced CD45RO expression, 16E8-F2 anti-CD45 MAb still detected a slight band at the position of recombinant CD45RO $(\sim 55 \mathrm{kDa})$ (Figure $5 \mathrm{~A}$ ). 


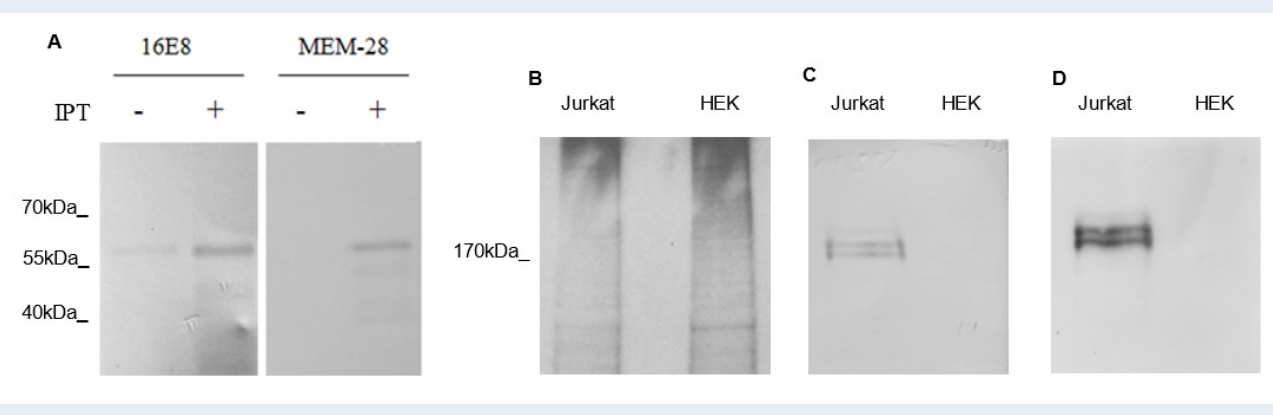

Figure 5: The activity of 16E8-F2 anti-CD45 MAb in Western blotting. (A): Western blotting analysis of recombinant CD45RO in the cell lysates of E.coli using 16E8-F2 anti-CD45 MAb and commercial MEM-28 anti-CD45 MAb; (B): SDS-PAGE analysis of Jurkat and HEK 293 cell lysates; (C): Western blotting analysis of Jurkat and HEK 293 cell lysates using MEM-28 anti-CD45 MAb and (D) using 16E8-F2 anti-CD45 MAb.

(A) HEK293- 16E8-F2
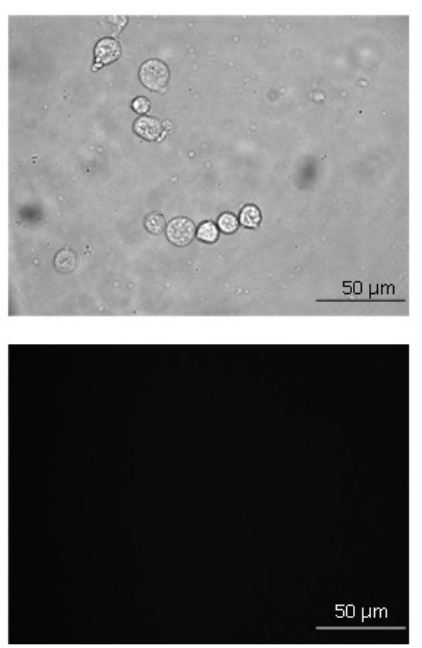

(B) Jurkat- MEM28
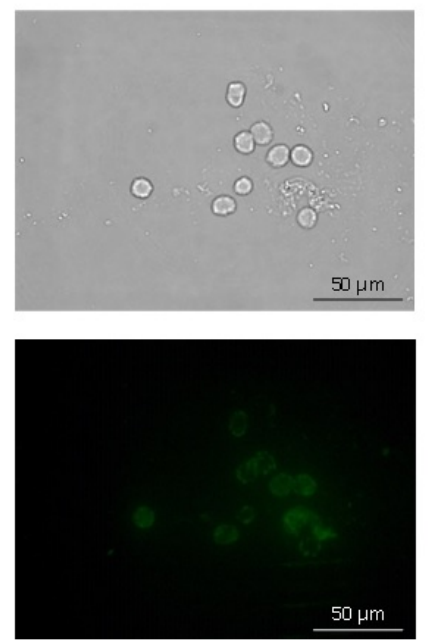

(C) Jurkat- 16E8-F2
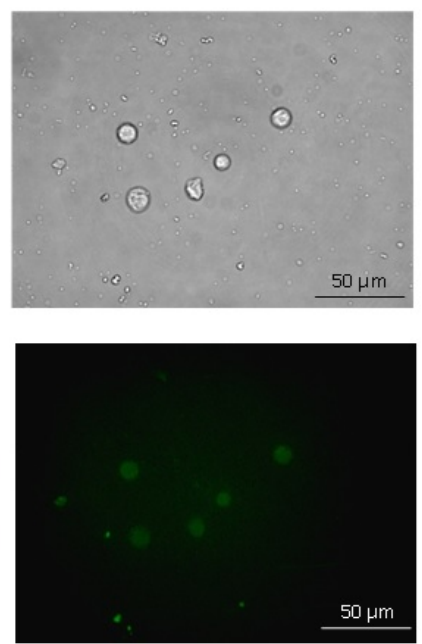

Figure 6: The activity of 16E9-F2 in fluorescent immuno-staining. (A): HEK293 cells stained with16E8-F2 antiCD45 MAb; (B): Jurkat cells stained with MEM-28 anti-CD45 MAb; (C): Jurkat cells stained with 16E8-F2 anti-CD45 MAb.

This band might be the recombinant CD45RO, which was expressed spontaneously by T7 lac operon in $E$. coli strain BL21 (DE3) in the absence of inducer IPTG. This phenomenon had been reported before ${ }^{8}$.

Besides CD45RO $(180 \mathrm{kDa})$ và CD45RA $(190 \mathrm{kDa})^{7}$, Jurkat $\mathrm{T}$ cells also express on their surface many CDs including CD18 (85 kDa), CD3 (23 kDa) ${ }^{9}, \mathrm{CD} 28$ (44 $\mathrm{kDa})^{10}, \mathrm{CD} 4(51 \mathrm{kDa})^{11}, \mathrm{CD} 5(54 \mathrm{kDa}), \mathrm{CD} 2$ (39 $\mathrm{kDa})^{12}$, CD6 $(72 \mathrm{kDa})^{13}$. Our results indicated that anti-CD45 MAb interacts specifically with CD45 antigen (180-190 kDa) in Western blotting. It neither cross-reacts with other CDs expressed on Jurkat $\mathrm{T}$ cells nor any proteins of non-leukocyte HEK293 cells. We used the extracellular region of C45RO isoform - the lowest molecular weight CD45 isoform- as the antigen to generate the monoclonal antibody. Logically, the antibody which can recognize CD45RO will also interact with all other CD45 isoforms, except the case that the epitope of antibody is located at the junction of exon 3 and 7 in CD45RO. In the Western blotting analysis with Jurkat $\mathrm{T}$ cell lysate, the 16E8-F2 anti-CD45 MAb detected a double band of protein at above $170 \mathrm{kDa}$. The double band may be CD45RO $(180 \mathrm{kDa})$ and CD45RA (190 kDa), which are simultaneously present on the Jurkat cell surface ${ }^{7}$. Since 16E8-F2 anti-CD45 MAb can interact with CD45RA, this antibody does not recognize the epitope located at the exon 3 and 7 junctions in CD45 protein. Therefore, it can be anticipated that the 16E8-F2 anti-CD45 MAb interacts with all isoforms of CD45. This an- 
ticipation is supported by the same result of Western blotting analysis using commercial MEM-28 antiCD45 MAb, which can interact with all CD45 isoforms according to the manual of the manufacturer. Nevertheless, the anticipation of specificity of 16E8F2 anti-CD45 MAb needs to be proved directly by further experiments.

\section{CONCLUSIONS}

$16 \mathrm{E} 8-\mathrm{F} 2$ anti-CD45 MAb created in this work is mouse $\operatorname{IgG} 2 \mathrm{~b} / \mathrm{k}$ isotype, it has a strong affinity with recombinant CD45RO antigen. The antibody may recognize specifically all isoforms of CD45. It can be used in ELISA, Western blotting, and fluorescent immuno-staining analyses. Our anti-CD45 MAb is potential for research and diagnosis applications. This is the first time an anti-humanCD45 monoclonal antibody was generated successfully in Vietnam, which might meet the domestic demands.

\section{AUTHORS' CONTRIBUTIONS}

Q.D.N. designed the research; G.H.T. and H.Q.N. performed research; Q.D.N., G.H.T. and Q.H.N. analyzed data; Q.D.N. wrote the paper. All authors read and approved the final manuscript.

\section{COMPETING INTERESTS}

The authors declare that they have no competing interests.

\section{ACKNOWLEDGEMENTS}

This project was funded by Biotechnology Center of Ho Chi Minh City. We acknowledge Assoc.Prof. Ho Huynh Thuy Duong and MSc. Tran Quoc Vu - Genetics Department, Biology and Biotechnology Faculty, University of Science, Vietnam National University Ho Chi Minh City - for their support in this research.

\section{REFERENCES}

1. Holmes N. CD45: all is not yet crystal clear. Immunology. 2006;117(2):145-155. PMID: 16423050. Available from: https: //doi.org/10.1111/j.1365-2567.2005.02265.x.
2. Tchilian EZ, Beverley PC. Altered CD45 expression and disease. Trends Immunol. 2006;27(3):146-153. PMID: 16423560. Available from: https://doi.org/10.1016/j.it.2006.01.001.

3. Penninger JM, Irie-Sasaki J, Sasaki T, Oliveira-dos-Santos AJ. CD45: new jobs for an old acquaintance. Nature immunology. 2001;2(5):389-396. PMID: 11323691. Available from: https://doi.org/10.1038/87687.

4. Lochem EG, Velden VHJ, Wind HK, Marvelde JG, Westerdaal NAC, Dongen JJM. Immunophenotypic Differentiation Patterns of Normal Hematopoiesis in Human Bone Marrow: Reference Patterns for Age-Related Changes and DiseaseInduced Shifts. Cytometry Part B (Clinical Cytometry). 2004;60B:1-13. PMID: 15221864. Available from: https://doi. org/10.1002/cyto.b.20008.

5. Gajendra S. Flowcytometry in Acute Leukemia. Clinics in Oncology. 2016;1:1166.

6. Beatty JD, Beatty BG, Vlahos WG. Measurement of monoclonal antibody affinity by non-competitive enzyme immunoassay. Journal of immunological methods. 1987;100(1-2):173-179. Available from: https://doi.org/10.1016/0022-1759(87)901876.

7. Lynch KW, Weiss A. A model system for activation-induced alternative splicing of CD45 pre-mRNA in T cells implicates protein kinase $C$ and Ras. Molecular and cellular biology. 2000;20(1):70-80. PMID: 10594010. Available from: https: //doi.org/10.1128/MCB.20.1.70-80.2000.

8. Briand L, Marcion G, Kriznik A, Heydel JM, Artur Y, Garrido C, Seigneuric R, Neiers F. A self-inducible heterologous protein expression system in Escherichia coli. Scientific Reports. 2016;6:33037. PMID: 27611846. Available from: https://doi. org/10.1038/srep33037.

9. Roose JP, Diehn M, Tomlinson MG, Lin J, Alizadeh AA, Botstein D, Brown PO, Weiss A. T Cell receptor-independent basal signaling via Erk and Abl kinases suppresses RAG gene expression. PLoS Biology. 2003;1(2):e53. PMID: 14624253. Available from: https://doi.org/10.1371/journal.pbio.0000053.

10. Freeman GJ, Gray GS, Gimmi CD, Lombard DB, Zhou LJ, White M, Fingeroth JD, Gribben JG, Nadler LM. Structure, expression, and $T$ cell costimulatory activity of the murine homologue of the human B lymphocyte activation antigen B7. Journal of Experimental Medicine. 1991;174(3):625-631. PMID: 1714935. Available from: https://doi.org/10.1084/jem.174.3.625.

11. Koka P, Yunis J, Passarelli AL, Dubey DP, Faller DV, Ynis EJ. Increased expression of CD4 molecules on Jurkat cells mediated by human immunodeficiency virus TAT protein. Journal of Virology. 1988;62(11):4353-4357. PMID: 2845147. Available from: https://doi.org/10.1128/JVI.62.11.4353-4357.1988.

12. Carmo AM, Castro MAA, Arosa FA. CD2 and CD3 associate independently with $C D 5$ and differentially regulate signaling through CD5 in Jurkat T cells. The Journal of Immunology. 1999;163(8):4238-4245.

13. Hassan NJ, Simmonds SJ, Clarkson NG, Hanrahan S, Puklavec MJ, Bomb M, Barclay AN, Brown MH. CD6 regulates T-Cell responses through activation-dependent recruitment of the positive regulator SLP-76. Molecular and cellular biology. 2006;26(17):6727-6738. PMID: 16914752. Available from: https://doi.org/10.1128/MCB.00688-06. 